\title{
La maternidad, \\ ¿un derecho de la mujer olvidado por FIFA?
}

\author{
Motherhood, a women's right \\ forgotten by FIFA?
}

José Alejandro Sierra Caballero²

\begin{abstract}
A pesar del reconocimiento de la maternidad dentro del catálogo de los derechos humanos reconocido por la legislación internacional, se encuentran protuberantes vacíos dentro de la normatividad FIFA que proteja eficazmente el derecho a la maternidad de las futbolistas profesionales. Por lo anterior se hace necesario que la FIFA otorgue mecanismos idóneos de protección al derecho de maternidad mediante regulación especial que proteja todo tipo de discriminación.
\end{abstract}

Palabras Clave: Maternidad, Derechos Humanos, FIFA.

1 Fecha de recepción: 12 de mayo de 2020. Fecha de aceptación: 26 de junio de 2020 Para citar el artículo: Sierra J. "La maternidad, cun derecho de la mujer olvidado por FIFA?". Revist@ E-Mercatoria,vol.19,n.ำ1, enero-junio, 2020.

DOI: https://doi.org/10.18601/16923960.v19n1.04

2 Abogado de la Universidad Santo Tomás de Aquino con especialización en Derecho Laboral y de la Seguridad Social de la Pontificia Universidad Javeriana y en Derecho Comercial de la Universidad de Los Andes. Adicionalmente, cuenta con estudios de Maestría en Asesoría Jurídica de Empresas del Instituto de Empresa de Madrid - España, en Derecho LLM. de la Universidad de los Andes y en International Sports Law del Instituto Superior de Derecho y Economía "ISDE" de Madrid - España. José Alejandro se ha desempeñado, entre otras cosas, como abogado asesor de la Oficina Jurídica del Ministerio de Agricultura, Coordinador del Área Laboral del Consultorio Jurídico de la Universidad de los Andes, en el año 2019 se convirtió en el primer colombiano en realizar prácticas jurídicas en el World Players' Union "FIFPRO" y en la actualidad, se desempeña como líder de la práctica en derecho deportivo de Brigard Urrutia Abogados. Correo electrónico: jsierra@bu.com.co. 


\begin{abstract}
Despite the recognition of motherhood within the catalog of human rights recognized by international law, there are protruding gaps within the FIFA regulations that effectively protect the right to maternity for professional soccer players. Therefore, it is necessary for FIFA to grant suitable protection mechanisms to the right of maternity through special regulations that protect all types of discrimination.
\end{abstract}

Key words: Motherhood, Human Rights, FIFA

\title{
INTRODUCCIÓN
}

Después de muchos años, una copa mundial de fútbol femenino fue bien recibida por el público en general, los resultados obtenidos en la última edición del año 2019 no tienen precedente alguno, teniendo en cuenta los informes relacionados con sus cifras ${ }^{3}$, principalmente, aquellas vinculadas con la cantidad de espectadores, el aumento de patrocinadores, el incremento de los beneficios destinados a los ganadores y evidentemente, la reacción de los seguidores en las redes sociales ${ }^{4}$, situación que permite reflejar de manera evidente, que el fútbol femenino puede ser considerado finalmente hoy en día, ya no solo como un proyecto a futuro sino como una realidad dentro de la industria del deporte.

A este respecto, el hecho de que el fútbol femenino adquiera cada vez más importancia dentro del negocio del fútbol nos permite preguntarnos por qué la Fédération Internationale de Football Association (en adelante, "FIFA") no ha incluido reglas específicas sobre los derechos de las mujeres y la eliminación de cualquier acto de discriminación en contra de ellas dentro de las relaciones contractuales, en especial, en aquellas vinculadas con la protección de la maternidad.

En este sentido, a través del presente artículo, me permitiré analizar como a pesar de la existencia de un compromiso expreso de la FIFA de promover, respetar y luchar por los derechos humanos alrededor del mundo, es posible evidenciar una impactante ausencia de normas vinculadas con la protección de la maternidad al interior de las regulaciones internacionales del fútbol. Con base en lo anterior, presentaré los fundamentos legales sobre los cuales

3 Morse, Ben, Article Women's World Cup in Numbers - Women's World Cup France 2019, Sports section, CNN, July 3, 2019, available on https://edition.cnn.com/2019/06/10/ football/womens-world-cup-statistics-spt-intl/index.html.

4 Sitio web de la FIFA, FIFA Women's World Cup 2019 watched by more than 1 billion, Article. 
considero que la protección de la maternidad debería incluirse de manera objetiva y formal en los reglamentos internacionales de la FIFA, como un principio de aplicación obligatoria por parte de todas las Federaciones locales, prosiguiendo con la exposición de mi propuesta de modificación de los principios emitidos por la FIFA sobre la duración y la terminación de los acuerdos laborales de las jugadoras de fútbol, para terminar más adelante con la presentación de las conclusiones correspondientes.

\section{FIFA Y LA PROTECCIÓN DE DERECHOS HUMANOS}

Para algunos puede resultar extraño o incluso absurdo establecer que la FIFA, entidad de carácter privado, se encuentra obligada a cumplir con los principios vinculados con la protección de los derechos humanos emitidos por organismos multilaterales, por cuanto, en principio, dicha obligatoriedad sólo recae en los Estados, los cuales, al ratificar dichas decisiones, estarán obligados a su acatamiento, sin embargo, antes de entrar en ese discernimiento, es absolutamente necesario describir aunque sea de una manera muy general a los organismos multilaterales vinculados con la promulgación de las normas internacionales de protección y desarrollo de los derechos humanos, al menos, aquellos vinculados en la protección de la maternidad en el ámbito laboral. Así, es imperativo traer a colación a las Naciones Unidas (en adelante, "ONU"), organización internacional creada por sus Estados signatarios el 26 de junio de $1945^{[5]}$, como la máxima autoridad mundial a cargo de, entre otros propósitos, reafirmar la fe en los derechos humanos fundamentales, la dignidad y el valor de la persona humana, en la igualdad de derechos de hombres y mujeres y de las naciones en general ${ }^{6}$. Actualmente la ONU está compuesta por 193 Estados Miembros ${ }^{7}$, y su sistema abarca la propia entidad y muchos programas afiliados, fondos y agencias especializadas, todos con su propia membresía, liderazgo y presupuesto ${ }^{8}$. Entre todas las agencias especializadas, es posible identificar a la Organización Internacional del Trabajo (en adelante, "OIT") a Gobiernos, empleadores y trabajadores para establecer normas laborales,

5 Sitio Web de la ONU, Carta de las Naciones Unidas del 26 de junio de 1945, Preámbulo, https://www.un.org/es/charter-united-nations/index.html.

6 Sitio Web de la ONU, Ibidem.

7 Sitio Web de la ONU, Estados Miembros, https://www.un.org/es/member-states/index. html.

8 Sitio Web de la ONU, Organización, Fondos Programas y Agencias, https://www.un.org/ es/sections/about-un/funds-programmes-specialized-agencies-and-others/index.html.

9 "La OIT fue creada en 1919, como parte del Tratado de Versalles que terminó con la Primera Guerra Mundial, y reflejó la convicción de que la justicia social es esencial para alcanzar una paz universal y permanente", Sitio Web de la OIT inicio, Acerca de la OIT, Historia de la OIT, https:// www.ilo.org/global/about-the-ilo/history/lang—es/index.htm. 
desarrollar políticas y diseñar programas que promuevan el trabajo decente para mujeres y hombres entre sus estados miembros ${ }^{10}$. Tanto la ONU como la OIT han alentado la protección de los derechos humanos en todo el mundo a través de sus recomendaciones y convenciones, que son vinculantes y exigibles para todos sus miembros.

Ahora, es muy importante enfatizar que, evidentemente, la FIFA no es un Estado miembro de la ONU o una entidad adscrita a la OIT, por lo tanto, en principio, los convenios internacionales no son vinculantes ni exigibles para esta organización. Sin embargo, y a pesar de esta situación, la FIFA, que es una asociación inscrita en el Registro Mercantil del Cantón de Zúrich de conformidad con el art. 60 ss. del Código Civil suizo ${ }^{11}$, encargada de controlar y gobernar el deporte del fútbol en todo el mundo, de conformidad con sus estatutos, se ha comprometido expresamente a respetar los derechos humanos y luchar por su protección en todo el mundo ${ }^{12}$.

Según lo anterior, está claro que la FIFA ha asumido la responsabilidad de luchar por los derechos humanos a nivel mundial dentro del espectro del fútbol, lo que obviamente incluye la incorporación en su marco legal de todos los estándares internacionales de derechos humanos establecidos por la propia onu, o cualquiera de sus agencias, como la OIT, persiguiendo en este sentido, la protección de todos los grupos vulnerables dentro de la industria del fútbol.

En este sentido, la FIFA, en cumplimiento de su compromiso expreso de respetar y luchar a favor de la protección de los derechos humanos, ha insistido en eliminar todo tipo de actos discriminatorios que puedan ejercerse en contra de cualquier individuo por motivos de raza, color de piel, origen étnico, nacional o social, género, discapacidad, orientación sexual, idioma, religión, opinión política, riqueza, nacimiento o cualquier otro estatus o cualquier otra razón ${ }^{13}$, ya sea porque este comportamiento haya sido ejercido por un individuo o contra cualquier persona o actor de la industria del fútbol, sin importar que esta provenga de dentro o fuera de su marco deportivo ${ }^{14}$. Además, la FIFA estableció un Consejo Asesor de Derechos Humanos en el año 2017, con el objeto de ayudar a fortalecer sus esfuerzos para garantizar el respeto de los derechos humanos. El Consejo está compuesto por ocho (8) expertos internacionales en derechos humanos, incluidas cuestiones laborales, de derechos y de lucha contra la corrupción, procedentes de Naciones Unidas, sindicatos, sociedad civil y empresas. Han sido nombrados por un periodo lang_es/index.htm.

11 Estatutos de la FIFA, Artículo 1, Edición 2019.

12 Artículo 3, Ibidem.

13 Artículo 4, Ibidem.

14 Código Disciplinario de la FIFA, Artículo 13, Edición 2019. 
inicial de dos (2) años. Esta organización proporciona a la FIFA asesoramiento y recomendaciones sobre todos los temas que considere relevantes para la implementación de sus responsabilidades en relación con su compromiso de respetar, promover y luchar por la protección de los derechos humanos en total consonancia con el artículo $3^{\circ}$ de sus Estatutos ${ }^{15}$.

Como podemos ver, la FIFA efectivamente ha asumido de manera importante su compromiso vinculado con ejercer todos los actos necesarios para proteger, promover y luchar por los derechos humanos en el mundo del fútbol, sin embargo, alcanzar este objetivo no es una tarea fácil y está lejos de completarse de manera efectiva, hasta tanto la FIFA tenga en cuenta a todos los grupos vulnerables que interactúan dentro de la industria del deporte que se encuentra bajo su dirección internacional, considerando que, en ocasiones, establecer un trato general igualitario dentro de la ley no es suficiente para evitar la ocurrencia de actos discriminatorios contra ciertos individuos, que históricamente han sido perseguidos o abusados por otros, como fue, por ejemplo debidamente entendido por la FIFA en el caso de menores, donde esta entidad protegió objetiva y específicamente sus derechos dentro de su marco lega ${ }^{16}$, pero que, en relación con otras personas, no se ha implementado ninguna protección específica, a pesar de las normas internacionales en materia de derechos humanos que se han consagrado en este sentido.

\section{PROTECCIÓN DE LA MATERNIDAD COMO DERECHO HUMANO}

La protección de los derechos de las mujeres ha sido un asunto prioritario para las Organizaciones Multilaterales de derechos humanos, en virtud a la determinación de la existencia de situaciones claras de persecución que sin razón justificada ha sufrido este grupo de personas en el curso de la historia, en donde, sus derechos han sido vulnerados en muchas ocasiones en función de su edad, origen étnico, nacionalidad, religión, estado de salud, estado civil, educación, discapacidad y estado socioeconómico ${ }^{17}$, entre otros motivos; por lo tanto, los derechos de las mujeres se han considerado como un tema de suma importancia al interior de la ONU, en donde, alcanzar una situación real de igualdad entre mujeres y hombres en todas las instancias de la vida

15 Sitio web de la FIFA, First Report by the FIFA Human Rights Advisory Board, Introduction, September 2017, https://es.fifa.com/search/?q=human+rights.

16 Reglamento sobre el Estatuto y Transferencia de Jugadores, Artículos 19 y 19bis, Edición 2019.

17 ONU, Women's Rights are Human Rights, United Nations Human Rights-Office of the High Commissioner, Introduction, Page 1, Publication New York and Geneva, 2014, available on https://www.ohchr.org/Documents/Events/WHRD/WomenRightsAreHR. pdf. 
política y social, y, además, eliminar todas las formas de discriminación, se ha considerado como un aspecto preponderante y dominante a la hora de analizar los escenarios de protección de los derechos humanos, y, a su vez, una parte clave de los valores de la organización ${ }^{18}$.

Entre las diferentes situaciones que deben estar vinculadas a la protección de los derechos de las mujeres, la maternidad ha surgido como uno de los temas más relevantes a tener en cuenta al momento de analizar las circunstancias de vulnerabilidad y abuso, debido a que este acontecimiento natural se ha utilizado como forma de discriminación en diferentes entornos, especialmente, en el ámbito laboral, donde las mujeres han tenido que soportar tratamientos injustos, principalmente relacionados con la imposición de barreras de contratación, la carga de condiciones vinculadas a su libre decisión de dar a luz y la imposibilidad de prestar servicios por un cierto período de tiempo ${ }^{19}$.

En este sentido la OIT, como una de las agencias adscritas a la ONU, no tardó mucho en emitir las primeras normas internacionales sobre protección de la maternidad, a través del Convención 3 de 1919 sobre Protección de la Maternidad, en donde, se emitieron normas en defensa de mujeres en estado de embarazo que prestaren sus servicios para empresas que desarrollan determinadas actividades, estableciéndose por primera vez la licencia de maternidad, un periodo de lactancia y la prohibición de finalizar el contrato de trabajo durante dicho periodo de licencia, es decir, la estabilidad laboral por maternidad ${ }^{20}$. Efectivamente, esta protección tenía una duración limitada en el tiempo, la cual, además, no era del todo clara, y, además, estaba supeditada a que el empleador tuviera conocimiento previo de la época del parto ${ }^{21}$. Desde cualquier punto de vista, esta regulación significó un cambio primordial a favor de la protección de los derechos de la mujer, sin embargo, no hay que dejar de lado que esta Convención fue emitida dentro del Tratado de Versalles, momento en el cual, el trabajo ejercido por parte de las mujeres, principalmente en las empresas denominadas como industriales era esencial para mantener la economía teniendo en cuenta la importante ausencia de mano de obra masculina una vez finalizada la primera guerra mundial ${ }^{22}$.

18 Ibidem

19 "a comprebensive system of protection to combat gender discrimination and to ensure equal opportunities and treatment between men and women in relation to their right to work by ensuring equal pay for work of equal value. In particular, pregnancies should not constitute an obstacle to employment and should not constitute justification for loss of employment.", Page 71, Ibidem.

20 Sitio Web de la OIT, Normas del Trabajo, Convención 3 de, 1919 (núm. 3). https://www. ilo.org/dyn/normlex/es/f?p=NORMLEXPUB:12100:0::NO::P12100 _ ILO _ CODE:C003.

21 Artículo 3, Ibidem.

22 Sitio Web de la OIT inicio, Acerca de la OIT, Historia de la OIT, https://www.ilo.org/ global/about-the-ilo/history/lang-es/index.htm. 
Los derechos de la mujer en el ámbito laboral fueron nuevamente analizados por parte de la OIT más de 30 años después, en donde, por medio del Convenio 103 sobre Protección de la Maternidad revisado en 1952 y vigente desde el 7 de septiembre de 1955, se aclararon algunas regulaciones de protección emitidas en la Convención 3 de 1919 y se otorgaron otras más, estableciéndose nuevamente cuales serían las ocupaciones laborales protegidas $^{23}$, el período de lactancia ${ }^{24}$, el cual fue tenido en cuenta como parte de la jornada laboral, el derecho a una licencia de maternidad vinculada a la existencia y notificación previa al empleador por parte de la trabajadora de un certificado médico que establezca la fecha presunta del parto ${ }^{25}$, y nuevamente, se consagró la protección reforzada por motivos de maternidad, por lo que se determinó que no era legal que el empleador notificara el despido durante la licencia de maternidad, o, que, informara a la trabajadora la terminación del contrato de tal suerte que la fecha de expiración del acuerdo de trabajo se diera durante el periodo de la licencia ${ }^{26}$.

Esta Convención también incluyó algunas disposiciones vinculadas con la posibilidad de que los miembros de la OIT pudieren presentar declaraciones relacionadas con la existencia de situaciones puntuales que pudieran configurar excepciones a la aplicación de las regulaciones, pero solo con respecto a ciertas ocupaciones ${ }^{27} \mathrm{y}$, además, se determinó que los principios preceptuados en la Convención solo serían vinculantes para los Estados cuyas ratificaciones se hayan registrado con su Director General ${ }^{28}$.

Teniendo en cuenta la fecha, desde mi punto de vista, la OIT dio un paso sorprendente para proteger los derechos de las mujeres y, sobre todo, en relación con el tema de este artículo, se le otorgó internacionalmente más fuerza a la prohibición de rescindir un contrato de trabajo por razones de maternidad.

Este movimiento internacional de reconocimiento de los derechos de las mujeres en el ámbito laboral trajo como resultado igualmente, que la protección de la maternidad fuera entendido como un derecho de carácter fundamental, considerando que todas las normas laborales consagradas en los Convenios y Recomendaciones de la OIT adquieren ese nivel, de conformidad con la Declaración de Filadelfia de 1944, por la cual, la comunidad internacional reconoció que el trabajo es parte de la vida diaria del individuo y es 1952 (núm. 103), Artículo 1, https://www.ilo.org/dyn/normlex/es/f?p=NORMLEXPUB:12 100:0::NO::P12100 _ ILO _ CODE:C103. 
crucial para la dignidad, el bienestar y el desarrollo de una persona como ser humano, por lo tanto, las normas internacionales del trabajo están ahí para garantizar que este propósito siga vigente para mejorar la vida y proteger la dignidad de hombres y mujeres en todo el mundo ${ }^{29}$.

A pesar de todo lo anterior, la protección de los derechos de las mujeres, y, en especial la protección a la maternidad en los entornos laborales, continuó siendo motivo de estudio y análisis por parte de la OIT, motivo por el cual, después de varios años, la Convención 103 de 1952 fue objeto de una segunda revisión por esta misma Agencia “... para promover aún más la igualdad de todas las mujeres en la fuerza laboral y la salud y seguridad de la madre y el niño, y para reconocer la diversidad en el desarrollo económico y social de los Miembros, así como la diversidad de las empresas y el desarrollo de la protección de la maternidad en la legislación y la práctica nacionales ... " ${ }^{30}$. En este sentido, la OIT procedió a emitir la Convención de Protección de la Maternidad 183 de 2000, a través de la cual, consagró nuevas normas laborales en defensa de los derechos de las mujeres extendiendo la protección para todas las industrias y teniendo en cuenta también a las trabajadoras embarazadas y lactantes, centrándose principalmente a la protección de su estado de salud, el desempeño laboral, la extensión de la duración de la licencia de maternidad, permisos en caso de enfermedad, beneficios médicos y financieros, implementación de sistema de seguridad social y, a los fines de este artículo, normas especiales de protección del empleo y no discriminación ${ }^{31}$.

En adición a las normas de protección emitidas por la OIT, es importante mencionar, que, la propia ONU, también ha consagrado normas en contra de los actos discriminatorios ejecutados contra las mujeres, entre los cuales es necesario mencionar la Convención sobre la eliminación de todas las formas de discriminación contra la mujer de 1979, presentado por esta Organización como un proyecto de ley internacional de derechos para las mujeres, que consta de un preámbulo y 30 artículos, por los cuales, principalmente, define la discriminación contra las mujeres ${ }^{32}$ y proporciona la base para lograr la igualdad entre mujeres y hombres garantizando a las primeras el mismo acceso y la igualdad de oportunidades en la vida política y pública, entre

29 OIT, Declaration of Philadelphia of 1944, Declaration concerning the aims and purposes of the International Labor Organization, Article 1, https://www.ilo.org/legacy/english/ inwork/cb-policy-guide/declarationofPhiladelphia1944.pdf.

30 Sitio Web de la OIT, C183-Convenio sobre la protección de la maternidad, 2000 (núm. 183), Preámbulo, https://www.ilo.org/dyn/normlex/es/f?p=NORMLEXPUB:12100:0::NO: :P12100 _ ILO _ CODE:C183.

31 Artículos 1 a 9, Ibidem.

32 Convention on the Elimination of All Forms of Discrimination against Women, United Nations Human Rights-Office of the High Commissioner, Part 1, Article 1, Publication New York, December 18, 1979, https://www.ohchr.org/en/professionalinterest/pages/ cedaw.aspx. 
los cuales, se incluyó específicamente el empleo ${ }^{33}$. Asimismo, los Estados Partes, acordaron tomar todas las medidas apropiadas, incluidas la legislación y las medidas especiales de carácter temporal, para que las mujeres puedan disfrutar de sus derechos humanos y libertades fundamentales ${ }^{34}$. Además, es relevante indicar que, considerando que esta disposición fue emitida por la ONU, sus principios y estándares de derechos constituyen un tratado de derechos humanos, por lo tanto, a partir de este momento, la maternidad fue considerada como un derecho humano de las mujeres, teniendo en cuenta que, de manera expresa, esta Convención incluyó a la maternidad como un derecho inherente a las mujeres como ser humano ${ }^{35}$.

Finalmente, considero muy importante traer a colación la publicación emitida por la ONU en el año 2014, denominada, "Los derechos de las mujeres son derechos bumanos", en la cual, la ONU expuso claramente que "... alcanzar la igualdad entre mujeres $y$ bombres y eliminar todas las formas de discriminación contra las mujeres son derechos bumanos fundamentales y valores de las Naciones Unidas ... " ${ }^{\prime 36}$. En este sentido, y considerando que la maternidad ha sido determinada como un derecho de la mujer por parte de la OIT, y, que, por este motivo, adquirió el carácter de derecho fundamental de acuerdo con los principios internacionales previamente mencionados en este escrito, asimismo, es muy importante indicar que, cuando la ONU clasifica como "Derechos Humanos" todas las normas internacionales dirigidas a evitar cualquier tipo de discriminación contra las mujeres, a partir de ese momento, igualmente, la maternidad fue catalogada por esta Organización como parte de los derechos humanos de las mujeres y por lo tanto, en un derecho que debe ser protegido y respetado en los términos concebidos por la OIT, como agencia de la ONU, por parte no solo de los Estados que hayan ratificado sus Convenciones en los términos de la ley internacional, sino también, por parte de aquellas organizaciones que de manera expresa y voluntaria se hayan obligado a lo mismo.

\section{PROTECCIÓN A LA MATERNIDAD Y LOS REGLAMENTOS DE LA FIFA}

Como se mencionó anteriormente, los derechos de las mujeres y, en particular, la maternidad fueron reconocidos internacionalmente por la ONU como parte de los derechos humanos, por lo tanto, la FIFA de conformidad con el artículo $3^{\circ}$ de sus Estatutos, está en la obligación de acatar todas las 
disposiciones emitidas por la ONU y sus agencias por tratarse de normas que desarrollan globalmente la protección de este tipo de derechos y, como se mencionó previamente, hacen al mismo tiempo parte de estos. En este sentido, la protección de la maternidad dentro de una relación laboral, como derecho fundamental y humano de la mujer, debería estar incluido formalmente en los reglamentos de la FIFA, teniendo en cuenta que esta organización es una entidad capaz de determinar sus propias reglas y que se ha comprometido de manera expresa y voluntaria a respetar, promover y luchar por la protección de los derechos humanos en el mundo del futbol. Esto es así, porque, la FIFA no puede asumir un compromiso expreso y formal dentro de sus Estatutos y figurar como un promotor global de la protección de los derechos humanos, para luego, internamente, negar este compromiso de manera injustificada al no aplicar las disposiciones relacionadas con la protección de la maternidad de acuerdo con los parámetros mínimos establecidos por las Convenciones de la ONU y la OIT.

A pesar de lo anterior, una protección formal de la maternidad dentro de las relaciones laborales en el mundo del fútbol no ha sido incluida por parte de la FIFA en alguno de sus reglamentos. Esta afirmación no proviene de una simple opinión, sino, por el contrario, de la revisión de cada uno de los reglamentos de la FIFA, en los cuales, no fue posible corroborar la inclusión de protección alguna en los términos establecidos por la OIT a través de sus convenciones. En este sentido, considero adecuado referirme a cada uno de los reglamentos de la FIFA, con el objetivo de confirmar mis afirmaciones.

\section{ESTATUTOS DE LA FIFA}

Considero oportuno dar inicio con una revisión de los Estatutos de la FIFA, es decir, el conjunto de normas legales por las que se regula el funcionamiento de la entidad, en donde básicamente, la FIFA fija sus objetivos, determina la composición de la entidad y su funcionamiento global, establece las reglas de las competiciones, medidas disciplinarias y las reglas del deporte, entre otras cosas, por lo que definitivamente se convierte en el cimiento normativo más importante de la entidad y sobre el cual la FIFA ejerce la dirección del fútbol alrededor del mundo ${ }^{37}$.

Es muy importante mencionar que los estatutos de la FIFA han incluido una disposición general en contra de la discriminación, por la cual, se prohíbe cualquier tipo de acto discriminatorio contra un país, persona privada o grupo de personas por motivos de raza, color de piel, etnia, nacional o social, género, discapacidad, idioma, religión, opinión, riqueza, nacimiento, orientación sexual o cualquier otra razón, lo cual, sitúa a la discriminación 
como un acto que atenta en contra de los principios de la organización y el desarrollo del deporte en todo el mundo ${ }^{38}$.

De esta forma, aunque se estableció de manera expresa la lucha en contra de la discriminación por parte de la FIFA en donde, específicamente, se prohíbe la realización de cualquier acto de carácter discriminatorio por razones de género, podría resultar comprensible que en algún momento se pudiera argumentar que con ocasión al hecho de que los derechos de la mujer se encuentran ubicados dentro de la protección general de género, estos, en especial la maternidad, es ya objeto de protección por parte de los Estatutos de la FIFA, sin embargo, este argumento resulta claramente alejado de las normas y recomendaciones de la ONU, en donde, se ha recomendado la implementación de una protección formal, clara y objetiva a favor de los derechos de las mujeres, en total oposición a la emisión de meras regulaciones generales que ignoran la necesidad de salvaguardar los derechos de estas personas de manera específica, en virtud de los años de abuso y persecución que este grupo de individuos ha tenido que soportar en el transcurso de la historia ${ }^{39}$.

Resulta igualmente difícil de entender, cómo a pesar de que uno de los objetivos principales de la FIFA está vinculado con el desarrollo del fútbol femenino ${ }^{40}$, esta entidad tampoco estableció una comisión encargada de velar por el cumplimiento de este objetivo ${ }^{41}$, situación que, en mi concepto, brindaría un respaldo muy importante a las mujeres que deseen ser jugadoras profesionales en aras de impulsar el desarrollo del fútbol femenino y otorgaría la posibilidad de que todos los temas vinculados con la necesidad de implementar medidas especiales para la protección de los derechos de las mujeres en la práctica del fútbol femenino sea discutido de manera formal e independiente ante el Consejo de la FIFA, en donde, expertos en el tema podrían asesorar y apoyar a este órgano de administración para proponer cambios esenciales en los reglamentos de la FIFA.

En este sentido, a pesar de que los estatutos incluyen de manera expresa como uno de sus principales objetivos el promover el desarrollo del fútbol femenino y la plena participación de las mujeres en todos los niveles de su gobierno, y, adicionalmente, se incluyó una prohibición general en contra de cualquier acto de discriminación, ni una palabra relacionada con la protección de los derechos de la mujer, en especial la maternidad en todos los ámbitos de desarrollo del deporte, a efectos de éste artículo en el ámbito laboral, fue incluida dentro de esta disposición.

Artículo 4, Ibidem.

Sitio Web de la ONU, Cuarta Conferencia Mundial sobre la Mujer, Beijing, 1995, https:// www.un.org/es/sections/issues-depth/gender-equality/index.html.

Estatutos de la FIFA, Artículo 2, Literal F, Edición 2019.

Capítulo V, Ibidem. 


\section{CÓDIGO DE ÉTICA}

Los estatutos de la FIFA no son el único reglamento de la organización que ha evitado incluir de manera expresa la protección del derecho a la maternidad en el ámbito laboral, considerando que tampoco ha sido incluida en el Código de Ética, reglamento que define los valores centrales más importantes para el comportamiento y la conducta de todos los individuos vinculados con la práctica del fútbol ${ }^{42}$. La protección de los derechos de las mujeres pasó desapercibida dentro de estas disposiciones y, una vez más, la FIFA sólo incluyó una disposición general en contra de la discriminación ${ }^{43}$, como si una disposición con tal redacción fuera suficiente para proteger a las mujeres de actos discriminatorios ejercidos en su contra por motivos de maternidad en el ámbito laboral.

Los estatutos de la FIFA establecieron la configuración de la Comisión Independiente de Ética, la cual según esta reglamentación llevará a cabo sus actividades y cumplirá con sus obligaciones de manera totalmente independiente, pero siempre defendiendo los intereses de la organización de conformidad con sus reglamentos. Esta Comisión ostenta el estatus de órgano judicial al interior de la organización y se rige por el Código de Ética de esta, teniendo entre otras facultades, aquellas vinculadas con el poder de sancionar a quien haya incurrido en alguna de las conductas establecidas como contrarias a la ética del deporte, y siempre que, la sanción esté debidamente consagrada en los reglamentos correspondientes ${ }^{44}$.

Evidentemente, este código determinó de manera expresa las conductas que son consideradas en contra de la ética de la organización y del fútbol, refiriéndose de manera concreta a cuáles actuaciones son consideradas como una vulneración de la integridad física y mental ${ }^{45}$, en donde, llama mucho la atención que los actos ejecutados en contra de la maternidad no hayan sido incluidos en ese listado, a pesar de que claramente, como ya se ha indicado en varias oportunidades en este escrito, cualquier acto ejecutado en contra de ese estado especial de salud de la persona ha sido entendido como un acto emprendido en contra de los derechos humanos de la mujer.

Adicionalmente, es importante resaltar que para la OIT el despido de una mujer embarazada, en licencia de maternidad o en periodo de lactancia, es considerado en sí mismo como un acto ilegal ${ }^{46}$ por lo que la jugadora que lo

42 Código de Ética de la FIFA, Preámbulo, Edición 2019.

43 Artículo 22, Ibidem.

44 Artículos 52 y 54, Ibidem.

45 Artículo 23, Ibidem.

46 Sitio Web de la oit, C183-Convenio sobre la protección de la maternidad, 2000 (núm. 183), Artículo 8, https://www.ilo.org/dyn/normlex/es/f?p=NORMLEXPUB:12100:0::NO: :P12100 _ ILO _ CODE:C183 
sufre no puede asumir en ningún caso la carga de la prueba para demostrar si existió o no una vulneración a su integridad física y mental para que sea considerada como un acto en contra de la ética de la FIFA, y por ende, su sola ocurrencia debería ser suficiente para que sea tomada como tal.

\section{CÓDIGO DISCIPLINARIO}

Este reglamento tiene como objetivo principalmente describir las infracciones y determinar las sanciones que estas conllevan, regular la organización y el funcionamiento de los órganos judiciales de la FIFA y establecer los procedimientos que deberán seguirse ante dichos órganos en el mundo del fútbol ${ }^{47}$. A través de esta regulación, la FIFA determina igualmente los sujetos pasivos de las sanciones que pueden ser implementadas, las cuales, están determinadas para ser impuestas a personas naturales y jurídicas, según sea el $\mathrm{caso}^{48}$.

Al igual que los reglamentos previamente analizados, este código establece una prohibición general de discriminación para señalar que cualquier acto de esa índole ejercido por uno de los sujetos pasivos de esta regulación será sancionado disciplinariamente por la autoridad correspondiente ${ }^{49}, \sin$ embargo, como ocurre en otras regulaciones de la FIFA, las conductas que puedan atentar en contra los derechos de la mujer, especialmente en contra de la maternidad, no fueron tenidas en cuenta como una infracción independiente dentro de su articulado.

De esta forma, es posible corroborar nuevamente que la FIFA prefiere mantener un criterio de protección general en contra de los actos de discriminación ejercidos en contra de la mujer, mencionando tan sólo la prohibición de cualquier acto discriminatorio por razones de género ${ }^{50}$, concepto absolutamente amplio que, por sí sólo, no permite evidenciar la existencia de los beneficios, protecciones y prohibiciones que han sido consagrados por parte de la OIT a favor del derecho a la maternidad en el ámbito laboral.

\section{REGLAMENTO SOBRE ESTATUTO Y TRANSFERENCIA DE JUGADORES "RETJ"}

Desde el punto de vista contractual, este es una de las regulaciones más importantes de la FIFA, en donde se determinan las normas mundiales y obligatorias concernientes al estatuto de los jugadores y su elegibilidad para 
participar en el fútbol organizado, así como su transferencia entre clubes de distintas asociaciones ${ }^{51}$.

El articulado inicial de este reglamento, concerniente a las definiciones, determina de manera expresa que todos los términos que se refieren a personas físicas se aplican indistintamente a hombres y a mujeres, preceptuando, además, que el uso del singular incluye también el plural y viceversa ${ }^{52}$, disponiendo de este modo, para todos los efectos, un tratamiento igual de carácter normativo del RETJ para mujeres y hombres, a lo cual, me surge de manera inmediata las siguientes inquietudes: ¿son realmente físicamente iguales? ¿se deben aplicar estas regulaciones de manera idéntica?

El derecho a la igualdad, el cual puede ser tenido en cuenta desde dos (2) perspectivas, es decir, aquel en donde todas las personas son iguales sea cual sea su origen, etnia, color, sexo, idioma, religión, opinión política o cualquier otra condición ${ }^{53}$, y adicionalmente, el otro, por el cual todas las personas son iguales ante la ley y tienen, sin distinción, derecho a igual protección por parte de la misma ${ }^{54}$, derechos, que, sin lugar a dudas, la FIFA ha introducido en los mismos términos dentro de sus regulaciones. A pesar de lo anterior, $\mathrm{y}_{\text {, }}$ como bien se mencionó previamente en este escrito, la forma de aplicación e implementación de los derechos humanos ha sido interpretada y desarrollada por la ONU y sus agencias, entre las cuales se encuentra la OIT, en donde, las mujeres han sido identificadas como un grupo objeto de persecución y abuso por parte de otros en el transcurso de la historia, por lo cual, la aplicación general del principio de igualdad no es suficiente para la protección de sus derechos, y por tanto, la implementación de normas explícitas que así la determinen son necesarias en el interior de las regulaciones de todos aquellos los países y organizaciones comprometidos con el respeto y protección de los derechos humanos ${ }^{55}$.

Predicar la igualdad de manera general en el fútbol entre mujeres y hombres es desconocer la naturaleza humana de cada uno de ellos y las situaciones de discriminación que con base en esa circunstancia las mujeres han tenido que padecer en el ámbito laboral, para efectos de este artículo, en donde la maternidad ha sido un criterio de trato diferencial absoluto que ha incidido en la decisión de muchos empleadores de contratar o no a la persona, en determinar sus actividades y en continuar o no con sus servicios, entre otras

51 Reglamento sobre el Estatuto y Transferencia de Jugadores, Artículo 1, Edición 2020.

52 "N. B. Todos los términos que se refieren a personas físicas se aplican indistintamente a bombres y a mujeres. El uso del singular incluye también el plural y viceversa", Definiciones, Ibidem.

53 Sitio web de la ONU, La Declaración Universal de Derechos Humanos, Artículo 1, https://www.un.org/es/universal-declaration-human-rights/.

54 Artículo 7, Ibidem

55 Sitio Web de la ONu, Cuarta Conferencia Mundial sobre la Mujer, Beijing, 1995, https:// www.un.org/es/sections/issues-depth/gender-equality/index.html. 
cosas, situaciones que evidentemente los hombres nunca han tenido que padecer, y que por tal motivo, trae como resultado el hecho de que la aplicación igualitaria de las normas laborales del RETJ no sean suficientes para proteger los derechos laborales de las jugadoras y si de vía libre a los empleadores a tomar decisiones totalmente contrarias a las normas internacionales de la OIT.

Resulta relevante mencionar, que, el único capítulo dedicado exclusivamente a cuestiones legales relacionadas con el fútbol femenino en este reglamento se encuentra vinculado únicamente a las normas de liberación de jugadoras para la participación en sus selecciones nacionales, descritos en el Párrafo 1bis del Anexo 1 del RETJ. No hay mención alguna al derecho de la maternidad.

Ahora, considerando que este escrito se enmarca en el contexto de la protección de la maternidad en el ámbito laboral, es importante destacar que a pesar de que esta protección se ha enmarcado como un derecho humano de la mujer, su inclusión no fue tenida en cuenta en el Artículo 1 del RETJ en donde este reglamento determinó las disposiciones internacionales de carácter vinculante a nivel nacional por cada federación, las cuales, por instrucción de esta normativa deben incluirse sin modificación alguna en sus reglamentos internos $^{56}$. A este respecto, la FIFA tuvo mucho cuidado de incluir en dichas disposiciones las relacionadas con el principio de estabilidad contractual, por el cual, el contrato de trabajo suscrito entre clubes y jugadores debe ser respetado en todo momento mientras este se encuentre vigente, estableciéndose igualmente las consecuencias legales derivadas de su terminación y la forma de llevarse a cabo con justa y sin justa causa, sin embargo ${ }^{57}$, no se tuvo en cuenta disposición alguna vinculada con la protección de la maternidad como una situación de trato especial al momento de configurarse cualquier forma de terminación de la relación laboral de una jugadora.

Esta circunstancia podría ser, para empezar, comprensible, teniendo en cuenta que no hay disposición alguna al interior del RETJ que haya regulado la protección de la maternidad como una forma de estabilidad laboral reforzada que impida la terminación del contrato de trabajo de las jugadoras por esa circunstancia, de acuerdo con los Convenios de la ONU y la OIT, y por el contrario, si revisamos el contenido y la redacción de los artículos 13 a 18 del RETJ, es posible corroborar que la FIFA no tuvo en cuenta la condición especial de maternidad de las mujeres al momento de establecer el principio de estabilidad contractual, las reglas vinculadas con la terminación de la relación laboral y las disposiciones especiales de los contratos de trabajo entre clubes y jugadores.

56 Reglamento sobre el Estatuto y Transferencia de Jugadores, Artículo 1, Numeral 3, Edición 2020.

57 Artículo 1, Numeral 3, Ibidem. 
En este sentido, nuevamente, la FIFA ha creado un marco legal en el que el principio de igualdad se aplica en términos generales, sin considerar a las mujeres como uno de los grupos de personas que merecen una protección formal y objetiva dentro de las regulaciones del fútbol, como ocurrió esencialmente con los menores, donde sus derechos y protecciones especiales están debidamente introducidas dentro de las regulaciones internacionales de fútbol ${ }^{58}$.

\section{OTRAS ESTRATEGIAS}

Finalmente, es importante tener en cuenta que la FIFA ha publicado la Estrategia de Fútbol Femenino mediante la cual describe cómo esta entidad trabajará con todas las partes interesadas para tomar medidas concretas con el fin de empoderar a las mujeres, hacer del fútbol un deporte para todos y luchar contra la discriminación por género. Sin embargo, a pesar de que la maternidad ha sido considerada como uno de los motivos más comunes de discriminación en el ámbito laboral, este asunto ni siquiera fue tocado por la publicación, donde, en ningún caso, la maternidad se consideró como una de las situaciones. que merece una protección especial dentro de la relación contractual existente entre clubes y jugadoras o, en cualquier caso, como una circunstancia que debe ser promovida por la FIFA para alentar el interés de las mujeres en la práctica del fútbol profesional.

En pocas palabras, la estrategia de impulso delineó diferentes tácticas vinculadas a escenarios en los que la FIFA trabajará para obtener la mejora de la práctica del fútbol femenino, sin embargo, pasó desapercibido todos los abusos laborales que las mujeres tuvieron que soportar durante décadas, y el hecho que para muchas mujeres, como se describirá más adelante, la práctica del fútbol podría estar encasillado como un factor en contra de su deseo de crear una familia, de acuerdo con la idea equivocada de que ser madre choca con el hermoso sueño de emprender una carrera como futbolista.

\section{CONCLUSIONES}

Como se mencionó anteriormente en este escrito, desde hace más de 100 años, la OIT estableció claramente una estabilidad laboral reforzada para las mujeres embarazadas, que, en caso de ser incorporada por la FIFA, cambiaría los principios internacionales del fútbol con respecto a la estabilidad contractual, la forma de terminación de la relación laboral, y efectivamente, las normas vinculadas con el término de duración de los contratos existentes entre clubes y jugadoras, considerando que, actualmente, estos contratos 
pueden rescindirse, ya sea, a su vencimiento, de manera unilateral con o sin justa causa por cualquiera de las partes o por mutuo acuerdo ${ }^{59}$, sin ningún tipo de estabilidad para el jugador una vez que la causa de terminación ha surgido legalmente.

A pesar de lo anterior, conforme a las normas vigentes de la OIT, la terminación del contrato de trabajo de una trabajadora durante la época de embarazo, licencia de maternidad o el periodo de lactancia es ilegal. De igual forma, será ilegal la notificación de la finalización del contrato de trabajo en aquellos casos en que el empleador lleve a cabo esa notificación durante la época de licencia de maternidad, o, que, en su caso, la notificación expire durante la licencia ${ }^{60}$.

En estos términos, si el club toma la decisión de culminar de manera unilateral con o sin justa causa el contrato de trabajo de una jugadora en estado de embarazo durante los periodos de estabilidad previamente señalados, esa terminación debería ser considerada ilegal y, por lo tanto, el acuerdo laboral se debería mantener hasta el día en que la jugadora retorne de su licencia de maternidad y culmine el periodo de lactancia correspondiente, y ya, solo en ese momento, el empleador tendría derecho a notificarle a la jugadora su decisión de finalizar el contrato de trabajo. Es muy importante mencionar que el convenio de la OIT no estableció nada sobre las terminaciones por mutuo acuerdo, por lo tanto, en principio, esa forma de terminación sería válida en cualquiera de los periodos de estabilidad reforzada, salvo que la autoridad correspondiente, en este caso la FIFA, establezca una disposición clara al respecto.

Las normas vigentes de protección internacional de los derechos de la mujer en el ámbito laboral, en especial de la maternidad, están contempladas en el Convenio 183 de 2000, por el cual, la OIT determinó una mayor protección laboral para las mujeres, al ampliar el alcance de la estabilidad para la maternidad establecida en el Convenio 103 de 1952, declarando que será ilegal que un empleador rescinda el contrato de trabajo de una mujer durante su embarazo o ausencia, ya sea en licencia por maternidad o enfermedad o durante un período posterior a su regreso al trabajo por lactancia, según lo prescriban las leyes nacionales, excepto por motivos no relacionados con el embarazo ${ }^{61}$.

Efectivamente, la carga de probar que los motivos del despido no están relacionados con el embarazo, el parto o la lactancia, recae en el empleador ${ }^{62}$,

Sitio Web de la OIT, C183-Convenio sobre la protección de la maternidad, 2000 (núm. 183), Artículo 8, https://www.ilo.org/dyn/normlex/es/f?p=NORMLEXPUB:12100:0::NO: :P12100 _ ILO _ CODE:C183.

61 Artículo 8, Ibidem.

62 Artículo 8, Numeral 1, Ibidem. 
por lo cual, la FIFA de conformidad con estas reglas, debe implementar no sólo los eventos de estabilidad laboral para las jugadoras, sino también, un proceso para revisar la validez de las terminaciones que se den en esos eventos por parte de los clubes. Además, el Convenio 183 de la OIT de 2000 consagró un período mínimo de licencia de maternidad de catorce (14) semanas, durante el cual, se debe garantizar un ingreso mensual para la trabajadora ${ }^{63}, \mathrm{y}$ también, el derecho de reincorporación al final de la licencia de maternidad, garantizándose que la mujer tenga derecho a volver al mismo puesto o un puesto equivalente una vez que finalice la licencia, manteniendo la misma remuneración previamente devengada ${ }^{64}$.

Además, y, para evitar cualquier comportamiento discriminatorio por parte del empleador, el Convenio 183 de 2000 también incluyó que se deben adoptar las medidas apropiadas para garantizar que la maternidad no constituya una fuente de discriminación en el empleo, por lo tanto, se estableció la prohibición de exigir una prueba de embarazo o un certificado de dicha prueba cuando una mujer esté en proceso de solicitud de empleo o trabajo, y así, esta circunstancia debe establecerse claramente dentro de las regulaciones laborales correspondientes, a menos que, con respecto a la actividad que se vaya a realizar, esto sea: (i) prohibido o restringido para embarazadas o lactantes mujeres bajo leyes o regulaciones nacionales; ${ }_{i}$ o (ii) cuando existe un riesgo reconocido o significativo para la salud de la mujer y el niño ${ }^{65}$.

En estos términos, la protección de la maternidad en el ámbito laboral ha sido desarrollada internacionalmente a través de convenciones en donde se han fijado disposiciones absolutamente claras de obligatoria aplicación por los países que las han ratificado y para aquellas organizaciones que como la FIFA se han comprometido formalmente a respetar, promover y luchar por la defensa de los derechos humanos.

\section{EN BUSCA DE UN CAMBIO}

Además de los comentarios efectuados a cada una de las regulaciones de la FIFA, en donde, normas específicas direccionadas a promover la protección de los derechos de la mujer, en especial de la maternidad, deberían ser promovidas e incorporadas por la FIFA, teniendo en cuenta el objetivo establecido en cada reglamento. En este punto, resulta claro igualmente, que las disposiciones internacionales de protección de la maternidad en el trabajo deben ser incluidas dentro de los principios globales que regulan las relaciones laborales contempladas en el RETJ, en donde, el derecho a la maternidad 
debería estar protegido de manera objetiva a través de reglas específicas de inclusión obligatoria dentro del marco legal de todas las federaciones locales.

A este respecto, $y$, siguiendo con las normas internacionales del trabajo de la OIT, el estado de embarazo, la licencia de maternidad y los períodos de lactancia deben ser incluidas como nuevas causas de restricción de la terminación del contrato de trabajo en el RETJ. En este sentido, estas reglas de protección deberían establecer, como mínimo, lo siguiente: (i) Derecho a la licencia de maternidad, incluso si las leyes laborales locales de una federación no la establecen, en cuyo caso, su duración debería ser establecida

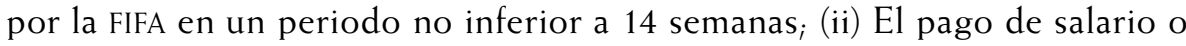
un porcentaje del mismo durante el periodo de licencia de maternidad $_{i}$ (iii) Garantizar el reintegro de la jugadora en las mismas condiciones laborales que tenía previo al inicio de la licencia; (iv) Derecho a un periodo de lactancia con posterioridad al reintegro; $(\mathrm{v})$ Ilegalidad de la terminación del contrato de trabajo de una jugadora durante el embarazo, licencia de maternidad y periodo de lactancia ("periodos de protección") $)_{i}$ (vi) La terminación del contrato de trabajo de la jugadora durante alguno de los periodos de protección sólo será permitida si esta provino de una circunstancia que no se encuentra relacionada con estos $;$ (vii) El Club que desee terminar el contrato de trabajo de una jugadora que se encuentre en alguno de los periodos de protección, deberá agotar un proceso especial de autorización ante la FIFA, (viii) La carga de probar que los motivos del despido no están relacionados con los periodos de protección recae en el empleador; (ix) Imponer sanciones económicas y disciplinarias a los clubes que desconozcan estos derechos, $\mathrm{y}_{i}$ (x) Adoptar medidas apropiadas para asegurar que la maternidad no constituya una fuente de discriminación en el empleo.

Para desarrollar estas propuestas, es importante resaltar que la incorporación de las reglas de protección de los derechos de la maternidad implica una participación real y activa de la FIFA en su compromiso de luchar por la protección de los derechos humanos en el fútbol. Obviamente, como ya mencioné antes, incluir estas disposiciones en el RETJ implica un gran cambio en los principios de estabilidad contractual de la FIFA, teniendo en cuenta que, por primera vez, se incluiría una prohibición para rescindir un contrato de trabajo dentro del mundo del fútbol, lo que a su vez constituiría el nacimiento del primer caso de estabilidad reforzada en los reglamentos internacionales de la organización.

Es importante mencionar, que, la autorización especial propuesta para dar por terminado el contrato de trabajo de una jugadora que se encuentre en alguno de los periodos de protección, debería ser incluido de manera formal en el RETJ, por tratarse del reglamento que consagra los principios del contrato de trabajo existente entre clubes y jugadores, en donde, además, la FIFA tendría que establecer el organismo a cargo de resolver este tipo de peticiones, el cual, por estar vinculado con temas de carácter laboral, lo más 
apropiado sería que este trámite de autorización fuere agotado ante la Cámara de Resolución de Disputas de la FIFA (en adelante "CRD"), entidad encargada de resolver las controversias laborales entre jugadores y equipos de fútbol ${ }^{66}$.

Igualmente, los periodos de protección de la maternidad en el ámbito laboral no pueden convertirse por sí mismos, y, bajo ningún motivo, en una causal de terminación del contrato de trabajo, por lo tanto, si un club desea rescindir el acuerdo laboral de una jugadora que se encuentra en alguno de los periodos de protección, este debe alegar una justa causa de terminación de conformidad con el artículo 14 del RETJ. Ahora, la discusión puede surgir si, como dije antes, el contrato de trabajo expira durante uno de los períodos de protección, caso en el cual, según los estándares internacionales de la OIT la terminación, sin excepción alguna, está prohibida y por tal motivo no tendría ninguna validez, por lo cual, este tipo de terminación natural de la relación laboral debería verse extendida hasta el momento en que finalice el periodo de lactancia.

Adicionalmente, la maternidad no debe ser una razón para que un club decida contratar o no contratar a una jugadora, teniendo en cuenta que, de conformidad con el Convenio 183 del 2000, las leyes locales deben establecer la prohibición de exigir una prueba de embarazo o un certificado de dicha prueba cuando una mujer se encuentre en proceso de selección. Ahora, teniendo en cuenta que el fútbol es un deporte de contacto, y que, por tal motivo, el estado de maternidad de la jugadora podría estar en riesgo en el evento en que el contrato se ejecute con normalidad, contar con esa información es absolutamente relevante pero sólo para adaptar el lugar de trabajo y el proceso de entrenamiento de la jugadora y no para definir su contratación.

En el mismo contexto y con el propósito de proteger internacionalmente los derechos de las jugadoras en los términos fijados por la OIT, es necesario que estas disposiciones sean incorporadas en el artículo 1 del RETJ, a fin de establecer que estas disposiciones sean vinculantes para todas las federaciones locales a nivel global y que deban incluirse sin ninguna modificación en sus reglamentos. Así, estaríamos frente a una verdadera protección internacional de los derechos de las jugadoras.

Por último, creo que el acatamiento de estas medidas requiere la inclusión de sanciones disciplinarias y económicas en los reglamentos de la FIFA, con el objetivo de que los clubes asuman la responsabilidad de vulnerar los derechos humanos de las jugadoras y en los mismos términos, con el propósito de que entiendan que estos derechos deben ser respetados con ocasión al compromiso asumido por la FIFA en sus estatutos ${ }^{67}$.

67 Estatutos de la FIFA, Artículo 3, Edición 2019. 


\section{ACTUALIDAD}

La situación actual para las mujeres en la industria del fútbol no es muy alentadora, por el contrario, de conformidad con el último Informe Mundial de Empleo FIFPRO 2017: Condiciones de Trabajo en el Fútbol Femenino profesional (en adelante, el "Informe"), solo el $2 \%$ de las encuestadas tienen hijos y el $47 \%$ tienen la seguridad de que abandonarían el juego para comenzar una familia, circunstancia que, en contraste con el hecho de que según el Informe, de los jugadoras que tenían hijos, el 61\% informó que no recibieron asistencia para el cuidado de los niños y, además, que los clubes o las federaciones nacionales proporcionaron el pago de maternidad solo al $8 \%$ de las encuestadas con hijos, y solo el $3 \%$ de los clubes brindaron apoyo, sin que se haya corroborado en algún caso que la federación local haya proporcionado apoyo para el cuidado de los niños a las jugadoras. Los resultados del Informe solo me permiten concluir que ignorar los derechos de la maternidad solo trae como resultado el desaliento de la práctica profesional del fútbol femenino y el fomento de tratos discriminatorios en el deporte.

Por otra parte, a pesar del hecho de que no hay decisiones emitidas por el Tribunal de Arbitramento Deportivo con respecto a la protección de la maternidad, hay casos impactantes en algunas federaciones alrededor del mundo, como el relacionado con la futbolista profesional de 24 años Rayveliz Hernández, en donde, la CRD de Venezuela dictaminó que el club, Flor de Patria, terminó legalmente el contrato de trabajo suscrito con la jugadora, la cual se encontraba en estado de embarazo, alegando que era "... indiscutible que debido a que la jugadora estaba embarazada, no podía cumplir con los términos de su contrato $y$, por lo tanto, el contrato debería considerarse rescindido"68, situación que claramente constituyó una violación de los derechos humanos de la jugadora y que permite evidenciar la forma en que las federaciones, clubes y tribunales locales desconocen los derechos de las futbolistas.

En pocas palabras, es incuestionable que las reglas de la FIFA deben enmendarse e introducir protecciones específicas para las jugadoras, no solo para desarrollar su participación en el fútbol profesional, sino con el propósito de adoptar los reglamentos de la organización a los criterios internacionales de la OIT y la ONU en relación con la protección de los derechos humanos alrededor del mundo en total consonancia con el compromiso asumido por la FIFA en este sentido a través de sus Estatutos. 


\section{BIBLIOGRAFÍA}

\section{ARTÍCULOS}

Morse, Ben, Article Women's World Cup in Numbers - Women's World Cup France 2019, Sports section, CNN, July 3, 2019.

Women's Rights are Human Rights, United Nations Human Rights-Office of the High Commissioner, Publication New York and Geneva, 2014.

\section{NORMAS}

Código de Ética de la FIFA, Edición 2019.

Código Disciplinario de la FIFA, Edición 2019.

Convention on the Elimination of All Forms of Discrimination against Women, United Nations Human Rights-Office of the High Commissioner, Publication New York, December 18, 1979.

Declaration of Philadelphia of 1944, Declaration concerning the aims and purposes of the International Labor Organization.

Estatutos de la FIFA, Edición 2019.

Reglamento sobre el Estatuto y Transferencia de Jugadores, Edición 2019.

\section{PÁGINAS DE INTERNET}

Sitio Web de la FIFA

First Report by the FIFA Human Rights Advisory Board, September 2017.

FIFA Women's World Cup 2019 ${ }^{\mathrm{TM}}$ watched by more than 1 billion, Article.

Women's Football Strategy.

Sitio Web de FIFPRO

Pregnancy in Women's Football, News \& Media.

Sitio web de la OIT

Acerca de la OIT.

Convención 3 de 1919. 
La maternidad, cun derecho de la mujer olvidado por FIFA?

Convención 103 de 1952.

Convención 183 de 2000.

Sitio web de la ONU

Carta de las Naciones Unidas del 26 de junio de 1945.

Estados Miembros

Organización, Fondos Programas y Agencias. 